\title{
MicroRNA-124 suppresses the invasion and proliferation of breast cancer cells by targeting TFAP4
}

\author{
NIER CHA ${ }^{1}$, BAOQING JIA ${ }^{1}$, YINZAI HE ${ }^{1}$, WEI LUAN ${ }^{2}$, WENHUA BAO $^{1}$, \\ XIUHUA HAN $^{1}$, WEISHI GAO ${ }^{1}$ and YANWEI GAO ${ }^{1}$ \\ Departments of ${ }^{1}$ Surgical Oncology and ${ }^{2}$ Medical Oncology, \\ Inner Mongolia People's Hospital, Hohhot, Inner Mongolia 010017, P.R. China
}

Received July 31, 2020; Accepted January 12, 2021

DOI: $10.3892 / \mathrm{ol} .2021 .12532$

\begin{abstract}
MicroRNA (miRNA/miR)-124 is widely accepted as the suppressor of different tumors. The present study aimed to improve understanding of the potential role of miR-124 in breast cancer. The gene expression profile change derived from the overexpression of miR-124 was investigated using RNA sequencing and bioinformatics analysis of the breast cancer cell line SKBR3. The results demonstrated that the gene expression profile of SKBR3 cells significantly changed. In addition, the transcription factor activating enhancer-binding protein 4 (TFAP4) gene was identified among the top 10 differentially expressed genes, and was identified as a novel target gene of miR-124 using a dual-luciferase reporter assay. TFAP4 knockdown in notably impaired SKBR3 cell migration and proliferation, which was consistent with decreasing migration and proliferation ability following overexpression of miR-124. Taken together, these results suggest that overexpression of miR-124 can suppress the migration and proliferation of SKBR3 cells by tarsgeting TFAP4. Thus, TFAP4 may act as a novel therapeutic target of breast cancer.
\end{abstract}

\section{Introduction}

Breast cancer is the leading cause of cancer-associated mortality (17-20\%) among women worldwide (1). Patients with breast cancer have a $41 \%$ 5-year survival rate, particularly those with metastatic disease (1). Despite improvement in the understanding of the pathogenic mechanisms involved in breast cancer, considerable challenges remain in the prevention and treatment of breast cancer.

MicroRNAs (miRNAs/miRs) are small non-coding RNA molecules that are 20-22 nucleotides in length (2). miRNAs

Correspondence to: Dr Yanwei Gao, Department of Surgical Oncology, Inner Mongolia People's Hospital, Hohhot, 20 Zhaowuda Road, Inner Mongolia 010017, P.R. China

E-mail: gaoyw0518@163.com

Key words: breast cancer, microRNA-124, transcription factor activating enhancer-binding protein 4 regulate various biological processes, including development, differentiation, apoptosis and proliferation, through imperfect pairing with target mRNAs of protein-coding genes and transcriptional or post-transcriptional regulation of their expression (3).

miRNAs have been proposed to contribute to oncogenesis since they can function as either tumor suppressors or oncogenes (3). For example, miR-124 functions as a tumor suppressor in the development of different tumors, such as glioblastoma (4), prostate cancer (5) gastric cancer (6) and lung cancer (7). In addition, miR-124 has been reported to inhibit the prognosis of patients with breast cancer by targeting several genes (8-16). Shi et al (8) demonstrated that STAT3 is a downstream target of miR-124, and STAT3 mRNA and protein downregulation was observed in breast cancer cells with upregulated miR-124 expression. STAT3 expression is downregulated following overexpression of miR-124, thereby inhibiting the proliferation and invasion of triple-negative breast cancer cells (8). Another study demonstrated that miR-124 can inhibit epithelial-to-mesenchymal transition (EMT) and metastasis of triple negative breast cancer cells by downregulating zinc finger E-box binding homeobox 2 expression (9). In addition, miR-124 can significantly inhibit the proliferation of breast cancer cells via cell cycle arrest, but does not induce apoptosis, by suppressing CD151 expression (10). Yan et al (11) identified $\alpha-1,6$-mannosylglycoprotein $6-\beta$-N-acetylglucosaminyltran sferase (MGAT5) as a target of miR-124, whereby miR-124 can suppress the proliferation and metastasis of breast cancer cells by regulating MGAT5 expression. In addition, several target genes of miR-124 have been identified in breast cancer, including snail family transcriptional repressor 2 (12), CBL (13), cyclin-dependent kinase 4 (14), flotillin-1 (15) and Ets-1 (16).

It has been reported that miR-124 regulates the expression levels of the aforementioned genes, resulting in inhibition proliferation and migration of breast cancer. In addition, clinical data have demonstrated that patients with breast cancer, with bone metastasis and high miR-124 expression have a longer survival time (17), whereas in vitro experiments suggest that cancer cell-derived miR-124 may inhibit the survival and differentiation of osteoclast progenitor cell by targeting interleukin 11 (17). 
Taken together, these findings suggest that miR-124 acts as both a key regulator of several oncogenes and a potential tumor suppressor in breast cancer. In addition, miR-124 is widely accepted as a negative regulator and inhibitor of tumor-derived cytokines, and abnormal miR-124 expression is associated with breast cancer progression (8-16). However, the molecular mechanism underlying the role of miR-124 in breast cancer, notably in Her2-positive/triple-negative breast cancer, is yet to be fully elucidated.

In the present study, miR-124 was overexpressed in Her2-positive breast cancer SKBR3 cells using lentiviral transduction. Subsequently, RNA sequencing was performed to investigate changes in gene expression profiles following overexpression of miR-124. The novel candidate miR-124 target genes were screened using bioinformatics analysis and the corresponding roles of these genes were investigated through proliferation and migration experiments. Thus, the present study aimed to improve our understanding of the potential role of miR-124 in breast cancer and to provide potential strategies of therapeutic intervention.

\section{Materials and methods}

Construction of the vector and stable cell line. The lentivirus with EGFP-expression miR-124 (lenti-miR-124) and negative control (lenti-NC) were purchased from Sangon Biotech, Co., Ltd. The SKBR3 breast cancer cell line was purchased from the American Type Culture Collection. A total of $2 \times 10^{-5}$ SKBR3 cells were seeded into 6-well plates and cultured in the DMEM (Thermo Fisher Scientific, Inc.) medium containing 10\% FBS (Gibco; Thermo Fisher Scientific, Inc.) at $37^{\circ} \mathrm{C}$ in the presence of $5 \% \mathrm{CO}_{2}$ for $12 \mathrm{~h}$.miR-124 precursorsequences were amplified and cloned into the lentiviral vector pCDH-CMV-MCS-EF1-copGFP (System Biosciences, LLC). A lentiviral vector that expressed GFP alone was used as a control. pCDH-miR-124 or control vectors $(6 \mu \mathrm{g})$ were co-transfected with the packaging plasmids psPAX2 $(4.5 \mu \mathrm{g})$ (System Biosciences, LLC) and pMD $(1.5 \mu \mathrm{g})$ (System Biosciences, LLC) into 3rd generation $293 \mathrm{~T}$ cells $\left(3 \times 10^{6}\right)$ using Lipofectamine $2000^{\circledR}$ (Invitrogen; Thermo Fisher Scientific, Inc.). After $48 \mathrm{~h}$ in $5 \% \mathrm{CO}_{2}$ at $37^{\circ} \mathrm{C}$, the virus-containing medium was harvested and subsequently pre-cleaned with a 3,000 x g centrifugation step and a $0.45 \mu \mathrm{m}$ filtration (Millipore; Merck KGaA). The virus-containing medium was overlaid on a sucrose-containing buffer $[50 \mathrm{mM}$ Tris- $\mathrm{HCl}$, pH 7.4,100 mM NaCl, $0.5 \mathrm{mM}$ ethylene diamine tetra acetic acid (EDTA)] at a 4:1v/v ratio and centrifuged at the indicated $9,000 \mathrm{xg}$ at $4^{\circ} \mathrm{C}$. After centrifugation, the supernatant was carefully removed and the tube was left standing for $3 \mathrm{~min}$. Phosphate buffered saline (PBS) was added to the semi-dried tube for re-suspension and then the tube was kept at $-80^{\circ} \mathrm{C}$ until further use. SKBR3 cells were subsequently infected with lenti-miR-124 or lenti-NC with $\mathrm{MOI}=1 \times 10^{-6}$ in the presence of $8 \mu \mathrm{g} / \mathrm{ml}$ polybrene (Chemicon International; Thermo Fisher Scientific, Inc.) Following transduction for 96 h, EGFP positive SKBR3 cells were selected using a FACS instrument (Celesta; $\mathrm{BD}$ Biosciences).The target cells were harvested for subsequent experimentation.

Total RNA extraction and sequencing. Total RNA was extracted from SKBR3 cells using the RNeasy Mini kit (cat. no. 74104; Qiagen, Inc.), according to the manufacturer's instructions. RNA Integrity Number (RIN) was measured using Agilent 2100 bio-analyzer. An RNA sequencing (seq) library was constructed using the Hieff NGSR MaxUP II DNA Library Prep kit for Illumina (cat. no. 12200E; Shanghai Yeasen Biotechnology Co., Ltd.), according to the manufacturer's instructions. Sequencing was performed by Novogene Biotech (www.novogene.com), using the HiSeq X Ten system (Illumina, Inc.) with HiSeq X Ten Reagent kit v.2.5 for 2x150 cycles (paired-end read length of $150 \mathrm{bp}$ ), 300 pM DNA was the loading concentration. Quality control was performed using Fastp and clean reads (reads contaminated by adaptors were removed, reads with Phred quality score $<5$ accounting for $>50 \%$ were removed, and reads with $\mathrm{N}$ content $>10 \%$ were removed) were mapped to the human genome (hg19) using TopHat software (v2.1.0) (18).

Reverse transcription-quantitative $(R T-q) P C R$. Total RNA was extracted from SKBR3 cells using the RNeasy Mini kit (cat. no. 74104 Qiagen, Inc.). RT of miR was performed using the One Step miR cDNA Synthesis kit (cat. no. D1801; Xinhai Gene Testing Co., Ltd.), and U6 was used as the internal reference for miR-124. The RT steps used were as follows: Initial denaturation step at $95^{\circ} \mathrm{C}$ for $30 \mathrm{sec}$, followed by 40 cycles of amplification at $95^{\circ} \mathrm{C}$ for $5 \mathrm{sec}$ and $60^{\circ} \mathrm{C}$ for $30 \mathrm{sec}$ using SYBR Green qPCR kit (cat. no. A2202A; Xinhai Gene Testing Co., Ltd.,). The relative transcript levels were quantified using the $2^{-\Delta \Delta \mathrm{Cq}}$ method (19) on the ABI 7500 (Applied Biosystems; Thermo Fisher Scientific, Inc.). The following primer sequences were used for qPCR: miR-124 forward, 5'-TAAGGCACGCGGTGAATGCC-3' and reverse, 5'-CAGGTCCAGTTTTTTTTTTTTTTTVN-3'; U6 forward, 5'-GCTTCGGCAGCACATATACTAAAAT-3' and reverse, 5'-CGCTTCACGAATTTGCGTGTCAT-3'. $\beta$-actin forward, 5'-AAAGACCTGTACGCCAACAC-3' and reverse, 5'-GTC ATACTCCTGCTTGCTGAT-3'; BCL6 forward, 5'-GAC TCTGAAGAGCCACCTG-3' and reverse, 5'-CTGGCTTTT GTGACGGAAAT-3'; IFR1 forward, 5'-ATGGCGACTAAG AAGCACAC-3' and reverse, 5'-CGAAGCCTGCTCATTGTA GT-3'; Mxd1 forward, 5'-TGAACATGGTTATGCCTCCA-3' and reverse, 5'-ACTTGATTCGGGTCCAAGTG-3'; LIF forward, 5'-TCTTGGCGGCAGGAGTTGTG-3' and reverse, 5'-CTTCTCCGTGCCGTTGGCGT-3'; and TFAP4 forward, 5'-GCAGGCAATCCAGCACAT-3' and reverse, 5'-GGAGGC GGTGTCAGAGGT-3'.

The following thermocycling conditions were used: Initial denaturation at $50^{\circ} \mathrm{C}$ for $2 \mathrm{~min}$ and $95^{\circ} \mathrm{C}$ for $5 \mathrm{~min}$, followed by 40 amplification cycles of $95^{\circ} \mathrm{C}$ for $20 \mathrm{sec}, 65^{\circ} \mathrm{C}$ for $10 \mathrm{sec}$ and $72^{\circ} \mathrm{C}$ for $30 \mathrm{sec}$ in the $\mathrm{ABI}$ instrument $(\mathrm{ABI})$.

Western blotting. SKBR3 were lysed in NP-40 Lysis-Buffer [150mMNaCl, $1 \% \mathrm{NP}-40$ and 50 mMTris (pH8.0)] and analyzed via BCA protein assay (cat. no. P0011; Beyotime Institute of Biotechnology). Proteins were denatured by heating for $5 \mathrm{~min}$ at $85^{\circ} \mathrm{C}$, separated via $10 \%$ SDS-PAGE (50 $\mu$ g protein/lane) and transferred to PVDF membranes (Merck KGaA). Membranes were blocked with $5 \%$ bovine serum albumin for $2 \mathrm{~h}$ at room temperature, and subsequently incubated with anti-TFAP4 antibody (1:1,000; cat. no. ab223771; Abcam) or anti-GAPDH (1:5,000; cat. no. bsm-33033M; BIOSS) overnight at $4^{\circ} \mathrm{C}$. 
After washing 3 times with PBS-Tween (0.1\% Tween-20), membranes were incubated with HRP-conjugated goat anti-rabbit IgG (1:10,000; cat. no. bs-0295G-HRP; BIOSS) at room temperature for $2 \mathrm{~h}$. Protein bands were visualized using the chemiluminescence kit (Thermo Fisher Scientific, Inc.).

Gene knockdown assay. Cells were seeded into 6-well plates and grown until $60 \%$ confluence. Cells were subsequently transfected with $50 \mathrm{nM}$ small interfering (si) RNA using Hiperfect reagent (Takara Bio, Inc.) in Opti-MEM medium (Thermo Fisher Scientific, Inc.). Transfected cells were cultured at $37^{\circ} \mathrm{C}$ in the presence of $5 \% \mathrm{CO}_{2}$ for $48 \mathrm{~h}$, after which subsequent experiments were performed. The TFAP4 targeting siRNA and non-target scramble controls were provided by Sangon Biotech Co., Ltd. (TFAP4 siRNA, 5'-GUG AUAGGAGGGCUCUGUAG-3'; and control 5'-GUAUCG GCUUAUCAGUCCGAGUAATT-3').

Report gene assay. The dual-luciferase reporter assay was performed, according to the manufacturer's instructions (cat. no. E1910; Promega Corporation). Briefly, the sequence wild type and mutated 3'-untranslated region (UTR) of TFAP4 was synthesized by Sangon Biotech, Co., Ltd., and subcloned downstream into the luciferase reporter gene pmirGLO vector (Promega Corporation). pmirGLO-3'-UTR-TFAP4 and the sequence of precursor miR-124 were subcloned into pSuper (OligoEngine), and the resulting pSuper-miR124, together with the reporter plasmid, were co-transfected into 293T cells using Lipofectamine ${ }^{\circledR} 3000$ (Thermo Fisher Scientific, Inc.). Following transfection and cultured at $37^{\circ} \mathrm{C}$ in the presence of $5 \% \mathrm{CO}_{2}$ for $48 \mathrm{~h}$, cells were harvested and lysed with $200 \mu \mathrm{l}$ Reporter Lysis Buffer (Promega Corporation). Luciferase activities were detected using the Luciferase Assay System (Promega Corporation). In this assay, the activity of firefly represented the experimental results and Renilla luciferase activity was used to normalize the data. The experiment was set up in triplicate and the experiment was carried out three times.

Cell invasion assay. The Transwell Matrigel ${ }^{\mathrm{TM}}$ assay was performed to assess cell invasion. Briefly, each well was coated with $60 \mu \mathrm{l} \mathrm{Matrigel} \mathrm{at} 37^{\circ} \mathrm{C}$ for $1 \mathrm{~h}$. A total of $200 \mu \mathrm{l}$ of DMEM medium without serum (Thermo Fisher Scientific, Inc.) containing $2 \times 10^{5}$ cells were plated in the upper chambers of 24-well Transwell plates with polycarbonate filters of $8-\mu \mathrm{m}$ pores (Corning, Inc.), while $600 \mu \mathrm{l}$ of DMEM medium (Thermo Fisher Scientific, Inc.) with $20 \%$ FBS was plated in the lower chambers. Following incubation at $37^{\circ} \mathrm{C}$ for $24 \mathrm{~h}$, cells in the upper chambers were removed using a cotton swab, and the migratory cells were fixed with $4 \%$ paraformaldehyde at $4^{\circ} \mathrm{C}$ for $1 \mathrm{~h}$ and stained with $2.5 \%$ crystal violet at room temperature for $1 \mathrm{~h}$. Stained cells were counted in six randomly selected fields using a light microscope (magnification, $\mathrm{x} 100)$.

Cell proliferation assay. The Cell Counting Kit-8 (CCK-8) assay (Takara Bio, Inc.) was performed to assess cell proliferation. For the CCK-8 assay, 5,000 cells were seeded into 96-well plates. At the 24, 48, 72 and $96 \mathrm{~h}, 10 \mu \mathrm{l}$ CCK-8 solution was added to each well and incubated for $4 \mathrm{~h}$ at $37^{\circ} \mathrm{C}$. Following incubation, cell proliferation was measured at a wavelength of $450 \mathrm{~nm}$.

Bioinformatics analysis. Cuffcompare v.0.8.3 was used to compare the similarity of transcripts and assess the construction of transcripts (20). Cuffmerge was used to combine multiple transcript sets into a single transcript set (20). Gene expression pattern was analyzed using PlotPCA package v1.12.3 (21) to reduce the dimensionality and a heatmap was constructed according to the gene expression using Pheatmap package v.1.0.10, (https://www.rdocumentation. org/packages/pheatmap/versions/1.0.10) in the R language. Cuffdiff was used to screen differentially expressed genes (DEGs) (20) and Ggplot2 package v.3.3.0 (https://www.rdocumentation.org/packages/ggplot2/versions/3.3.0) was used to construct the volcano plot. The distribution of DEGs was mapped on chromosomes using graphics function of R language. Gene Ontology (GO) enrichment analysis of DEGs was performed using Clusterprofiler package v.3.0.2, (https://www. rdocumentation.org/packages/clusterProfiler/versions/3.0.2) of $\mathrm{R}$ language. The similarities between GO terms were calculated using GOSemSim v.1.28.1, (https://www.rdocumentation. org/packages/GOSemSim/versions/1.28.1) and GO terms cluster was labeled using Ggtree v.1.4.11, (https://www. rdocumentation.org/packages/ggtree/versions/1.4.11). The potential downstream genes of miR-124 was predicted using the TargetScan database v.7.2 (http://www.targetscan. org/vert_72/). Combined with the DEGs from RNA-seq, the intersection was used for semantic similarity analysis using GOSemSim. The Protein-protein interaction network was analyzed using the Search Tool for the Retrieval of Interacting Genes/Proteins (STRING) database (https://string-db.org). The threshold for screening DEGs was adjusted $\mathrm{P}<0.05$, and the multiple difference was $>2$-fold. Adjusted P-value $<0.05$ and gene counts $>5$ were used as thresholds to identify significant $\mathrm{GO}$ terms.

Ethics. Although human samples or patient data were not included in the present study, CNSA requires ethics approval when uploading sequencing data. The present study was approved by the Ethics Committee of Inner Mongolia Autonomous Region People's Hospital, (Hohhot, China) (approval no. 202001005L).

Statistical analysis. GraphPad Prism 8.0 (GraphPad Software Inc.) was used for statistical analysis. All experiments were performed in triplicate and data are presented as the mean \pm standard deviation. unpaired Student's t-test was used to compare differences between two groups, while one-way ANOVA and Bonferroni post hoc test (non-parametric test) were used to compare differences between multiple groups. $\mathrm{P}<0.05$ was considered to indicate a statistically significant difference.

\section{Results}

Overexpression of miR-124 suppresses the migration and proliferation of SKBR3 cells. To determine the role of miR-124 in the pathogenesis of Her2-positive breast cancer, miR-124 was overexpressed in SKBR3 cells using lentivirus 

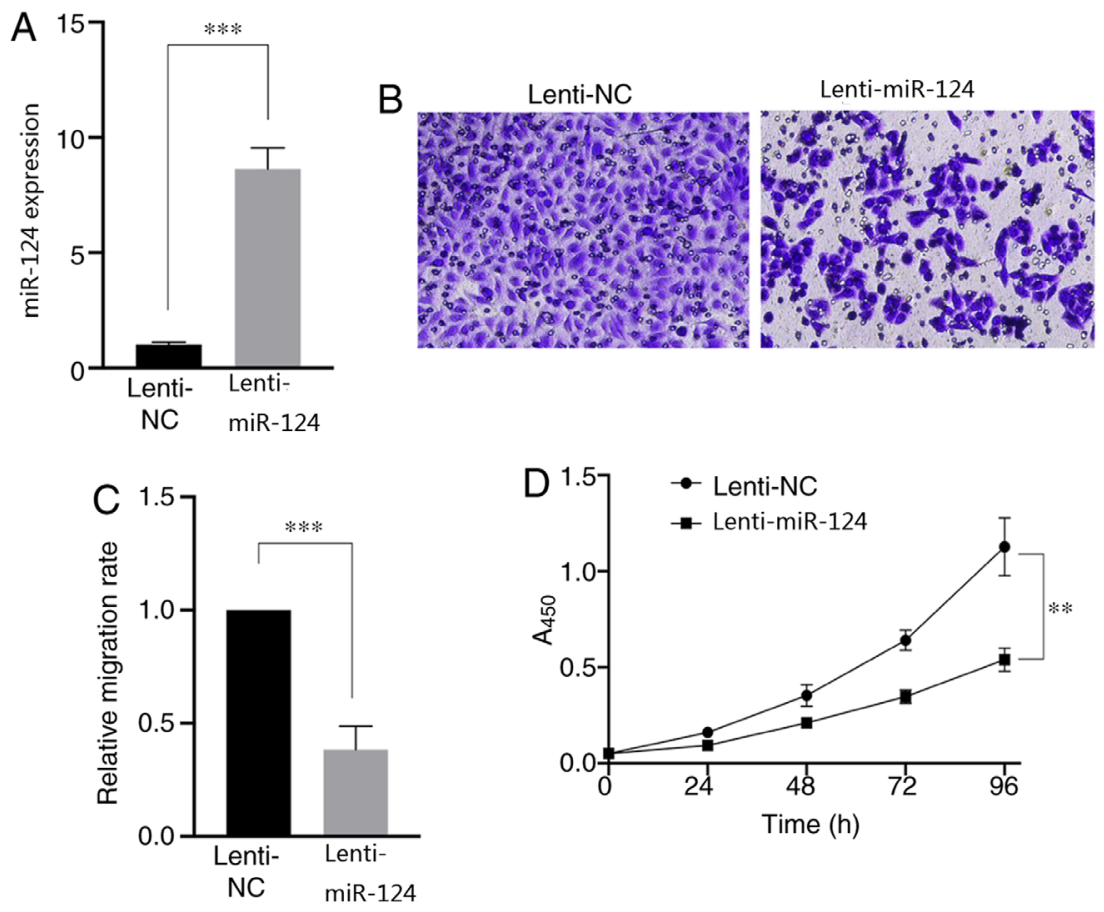

Figure 1. Effects of miR-124 overexpression in SKBR3 cells. (A) miR-124 expression in lenti-miR-124 infected SKBR3 cells and control cells. (B and C) The Transwell assay was performed to assess the effect of overexpression of miR-124 on the migratory ability of SKBR3 cells. (D) The Cell Counting Kit- 8 assay was performed to assess the effect of overexpression of miR-124 on the proliferative ability of SKBR3 cells. ${ }^{* *} \mathrm{P}<0.01,{ }^{* * * *} \mathrm{P}<0.001$. miR, microRNA; NC, negative control.

and RT-qPCR analysis was performed to assess transfection efficiency (Fig. 1A). Subsequently, cell migration and proliferation assays were performed to assess the change in cell malignancy following overexpression of miR-124. The results demonstrated that overexpression of miR-124 significantly impaired cell migration $(\mathrm{P}<0.001$; Fig. $1 \mathrm{~B}$ and $\mathrm{C})$ and suppressed cell proliferation (Fig. 1D). Taken together, these results suggest that overexpression of miR-124 can significantly influence the migratory and proliferative abilities of SKBR3 cells $(\mathrm{P}<0.01)$. Thus, total RNA was extracted from SKBR3 cells overexpressed with miR-124 for sequencing.

Quality evaluation of sequencing data. In the present study, the PlotPCA package was used to assess the transcriptome sequencing data of SKBR3 cells overexpressed with miR-124 and control cells. Following dimensionality reduction, the two principal components, $\mathrm{PC} 1$ and $\mathrm{PC} 2$, can explain $>80 \%$ of the sample differences, which demonstrated that $\mathrm{PC} 1$ and PC2 were sufficient for cluster analysis of the differences in miR-124 expression (Fig. 2A). Since the transcriptome data of the same processed samples should have had the same characteristics, the principal components, $\mathrm{PC} 1$ and $\mathrm{PC} 2$, were used to depict the expression characteristics of different samples. The results demonstrated that both SKBR3 cells overexpressed with miR-124 and control cells exhibited high similarity; however, the expression characteristics of the two groups of samples were different (Fig. 2B).

DEGs. Raw reads contaminated by adaptors were removed, reads with Phred quality score $<5$ accounting for $>50 \%$ were removed, and reads with $\mathrm{N}$ content $>10 \%$ were removed. Clean reads were mapped to the reference to calculate the expression value $\mathrm{o}$. The expression value was normalized by sequencing depth to ensure the expression levels of all genes were comparable across samples, which indicated the quality of the original data (Fig. 2C). Hierarchical cluster analysis exhibited the comprehensive DEG patterns of samples with miR-124 overexpression and miR-NC. The effect of miR-124 on DEGs in SKBR3 cells further supported the role of miR-124 in breast cancer (Fig. 2D). Differential expression analysis was performed with all the genes and 716 DEGs were identified between the control group and the miR-124 overexpression group. Thus, PCR analysis was subsequently performed to verify the results following bioinformatic analysis. Among the 717 DEGs, 418 genes were upregulated and 299 genes were downregulated following overexpression of miR-124 (Fig. 2E). The detailed list of gene expression is presented in Table SI.

miR-124 associated pathway and genes analysis. GO enrichment analysis was performed on the DEGs. Using the corrected P-value as the threshold, a total of $42 \mathrm{GO}$ pathways were identified (Table SII), of which the top ten pathways were 'maintenance of location', 'regulation of intracellular protein transport', 'regulation of MAP kinase activity', 'semi-lunar valve development', 'cellular response to biotic stimulus', 'cell cycle arrest', 'cell junction organization', 'DNA damage checkpoint', 'astrocyte activation' and 'negative regulation of myoblast differentiation' (Fig. 3A). Given that miRNAs function to degrade the target gene RNA (3), genes with cells expressing low levels of miR-124 and genes that overlapped with target genes regulated by miR-124, which was predicted using the TargetScan database (http://www.targetscan. org/vert_72/), were selected. To determine the genes most 

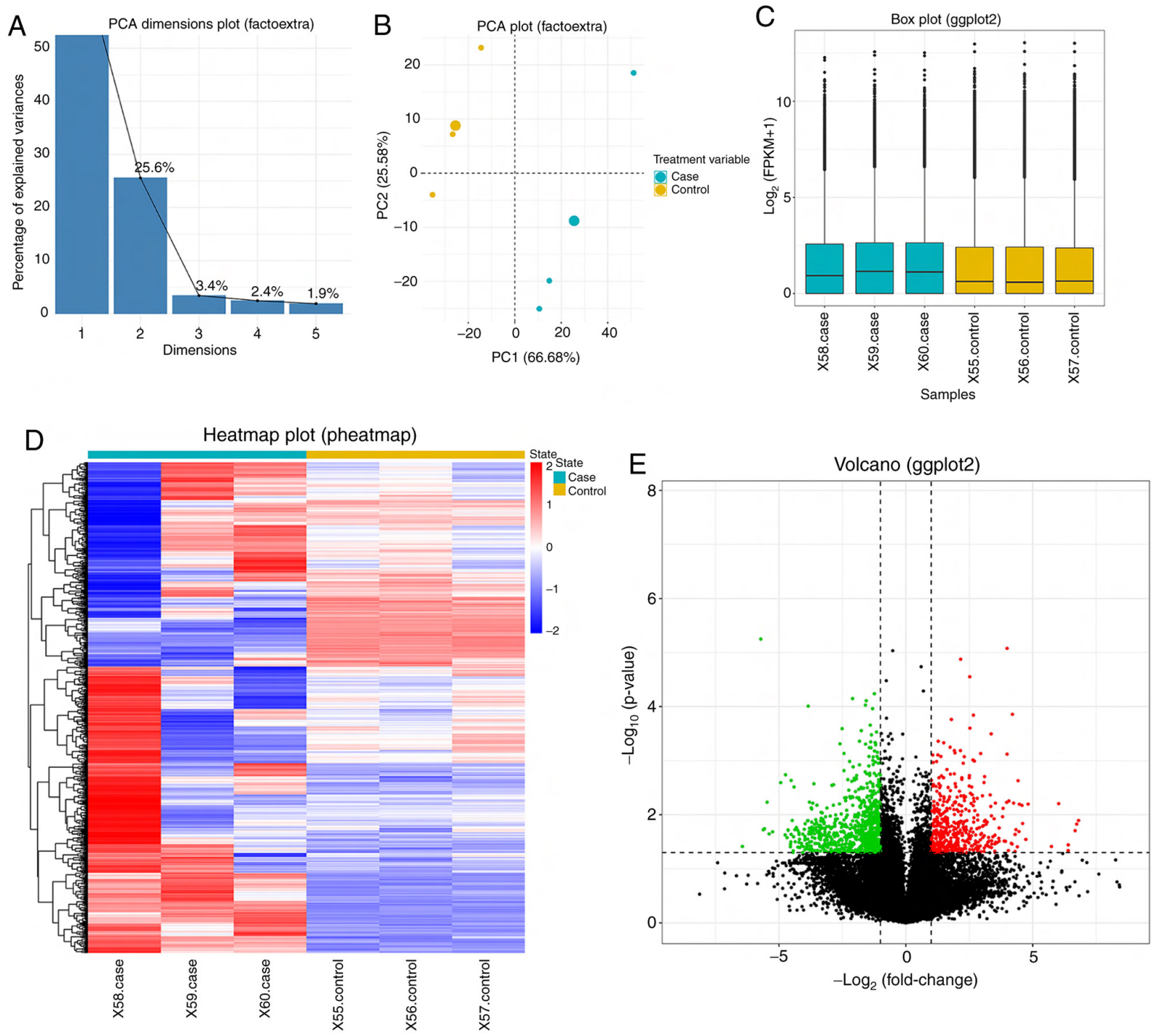

Figure 2. Transcriptome profile of SKBR3 cells with high and low miR-124 expression. (A) The histogram presents the contribution rate of each principal component following dimensionality reduction by PCA. (B) The sequencing data between different samples was visualized using PC1 as the abscissa and PC2 as the ordinate. The blue dots represent cells overexpressing microRNA-124 and the yellow dots represent control cells. (C) Box plots present the normalization of expression between samples. The blue boxes represent cells overexpressing miR-124 and the yellow boxes represent control cells. (D) Heat map exhibits differences in gene expression between different samples. (E) Volcano plot exhibits differentially expressed genes. The red dots represent upregulated genes, while the green dots represent downregulated genes. The black dots represent non-differential expression genes.

closely associated with miR-124 in SKBR3 cells in this list, the average functional similarity associations of the genes were calculated. The results demonstrated that the four genes with the highest scores were interferon regulatory factor 1 (IRF1), B-cell lymphoma 6 (BCL6), MXD1 and leukemia inhibitory factor (LIF) (Fig. 3B). Notably, although the negative regulation of myoblast differentiation and astrocyte activation pathways in the GO enrichment analysis had the largest proportion of genes downregulated by miR-124, the two most important genes identified in functional similarity analysis, IRF1 and LIF genes, were mainly enriched in the 'cell cycle arrest' term.

TFAP4 expression is regulated by $m i R-124$. The dual-luciferase reporter assay was performed to determine whether TFAP4 was directly regulated by miR-124 (Fig. 4A). The overexpression of miR-124 significantly decreased the relative luciferase activity of cells transfected with 3'-UTR of the TFAP4, the results of
RT-qPCR and western blot analyses demonstrated decreased mRNA and protein levels of TFAP4 (Fig. 4B and C). In addition, TFAP4 expression significantly decreased following overexpression of miR-124 (Fig. 4D), suggesting that miR-124 inversely regulates TFAP4 expression.

Downregulation of TFAP4 attenuates the migratory ability of SKBR3 cells. To further confirm that downregulation of TFAP4 can affect the migratory ability of SKBR3 cells, cells were transfected with si-TFAP4. As presented in Fig. 5A and B, TFAP4 mRNA and protein expression levels were significantly downregulated in SKBR3 cells following TFAP4 knockdown compared with the control. As presented in Fig. 5C and D, the migratory ability of SKBR3 cells was significantly reduced following TFAP4 knockdown compared with the control samples. Taken together, these results suggest that downregulation of TFAP4 significantly impairs the migratory ability of SKBR3 cells. 

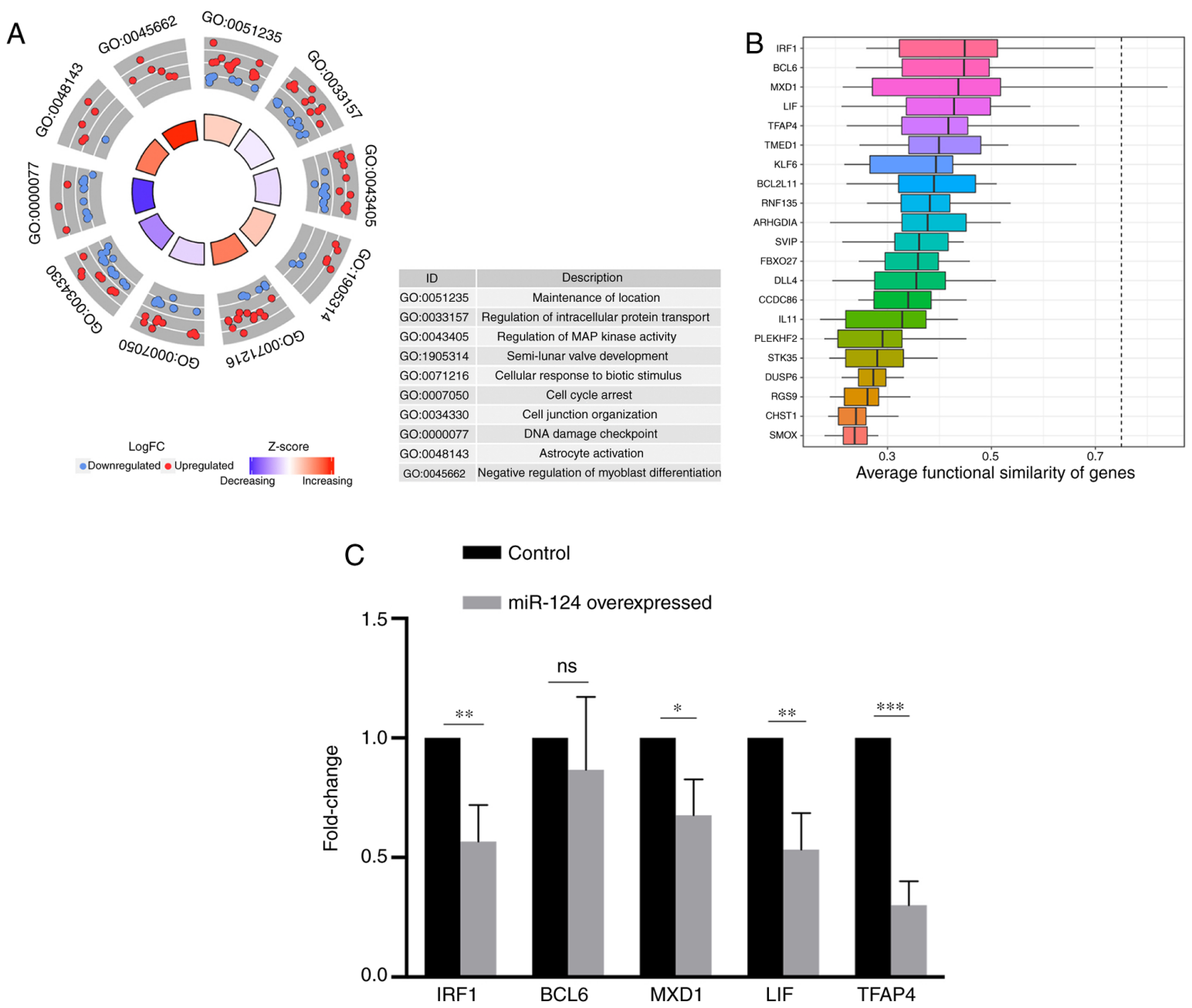

Figure 3. GO analysis of differential expression genes and PCR verification. (A) GO analysis was performed to determine the GO terms with the highest degree of enrichment. The red dots represent upregulated genes and the blue dots represent downregulated genes in the respective terms. (B) Box plots represent the semantic similarity of potential targeted genes of miR-124. (C) Reverse transcription-quantitative PCR analysis was performed to detect the expression levels of the top five differentially expressed genes in SKBR3 cells overexpressed with miR-124. ${ }^{*} \mathrm{P}<0.05,{ }^{* *} \mathrm{P}<0.01,{ }^{* * *} \mathrm{P}<0.001 \mathrm{GO}$, gene ontology; miR, microRNA.

Downregulation of TFAP4 suppresses the proliferation of SKBR3 cells. The CCK-8 assay was performed to assess the effect of downregulating TFAP4 expression on the proliferation of SKBR3 cells. As presented in Fig. 6, transfection with si-TFAP4 significantly decreased the proliferative ability of SKBR3 cells compared with the control group, which is consistent with the results following overexpression of miR-124 in SKBR3 cell (Fig. 1D). Taken together, these results suggest that TFAP4 knockdown significantly decreases the proliferative ability of SKBR3 cells.

\section{Discussion}

miR-124 targets several genes, such as STAT3, CD151 and MGAT5 ( $\alpha-1,6$-mannosylglycoprotein $6-\beta-\mathrm{N}$-acetylgluco saminyltransferase) (8-16); thus, the present study aimed to investigate changes in malignancy in SKBR3 cells and screen potential novel target genes for miR-124. Thus, miR-124 was overexpressed in SKBR3 cells. By sequencing the transcriptome of the transfected cells, it was determined that overexpression of miR-124 significantly changed gene expression in SKBR3 cells compared with the control group, and ultimately decreased the malignancy of tumor cells, which was consistent with the findings by Lin et al (22). In addition, the results of the present study demonstrated that miR-124 negatively regulated the expression of the TFAP4 gene, and downregulation of TFAP4 expression significantly impaired the migratory and proliferative abilities of SKBR3 cells.

The TFAP4 gene is a member basic helix-loop-helix leucine-zipper domain family (23) and participates in the regulation of cell proliferation and differentiation, metastasis, angiogenesis, as well as other biological functions in tumors (24). Overexpression of TFAP4 is associated with unfavorable prognosis of patients with gastric cancer (25), colorectal cancer (24), prostate cancer (26) and non-small cell lung carcinoma (27). Mechanistically, Huang et al (28) demonstrated that TFAP4 can promote hepatocellular carcinoma invasion and metastasis by activating the PI3K/AKT signaling pathway (28), EMT (29) and the Wnt/ $\beta$-catenin pathway (30). It has also been reported that abnormal degradation of TFAP4 can block cell mitosis, leading to the activation of the DNA damage response (31). This finding is consistent with the results of the present study, where TFAP4 knockdown blocked 


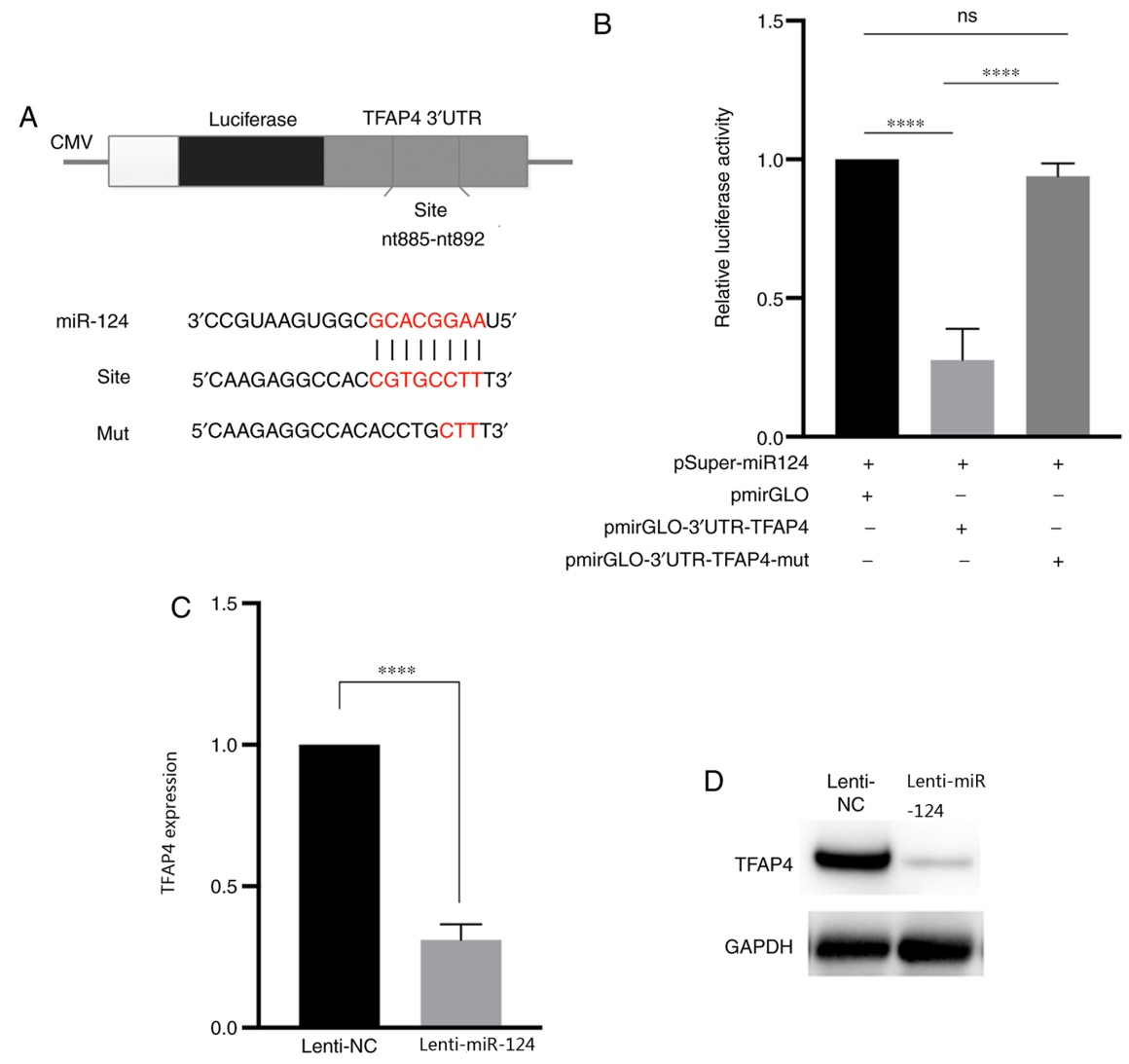

Figure 4. miR-124 directly targets TFAP4 in SKBR3 cells. (A) Binding site of the wild-type and mut TFAP4 3'-UTR (mut site is labeled in lowercase letters and underlined). (B) The dual-luciferase reporter assay was performed to assess the effect of miR-124 on the luciferase activity of 3'-UTR of TFAP4. Data are presented as the mean of three independent experiments. (C and D) Overexpression of miR-124 significantly downregulated TFAP4 expression. ${ }^{* * * *} \mathrm{P}<0.0001$. miR, microRNA; TFAP4, transcription factor activating enhancer-binding protein 4; mut, mutant; UTR, untranslated region; ns, no significance.
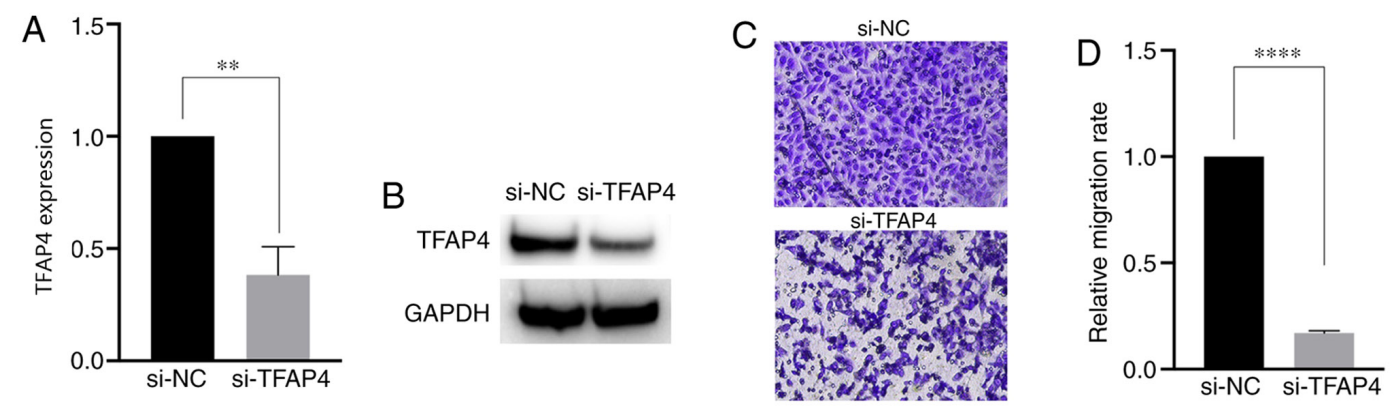

Figure 5. TFAP4 knockdown significantly impairs the migratory ability of SKBR3 cell. (A) Reverse transcription-quantitative PCR and (B) western blot analyses were performed to assess TFAP4 knockdown efficiency. (C) Representative images and (D) quantification analysis of the Transwell assay demonstrated that transfection with si-TFAP4 significantly impaired the migratory ability of SKBR3 cells. Cells were seeded into a Matrigel precoated Transwell membrane. ${ }^{* * *} \mathrm{P}<0.01,{ }^{* * * *} \mathrm{P}<0.001$. TFAP4, transcription factor activating enhancer-binding protein 4 ; si, small interfering; NC, negative control.

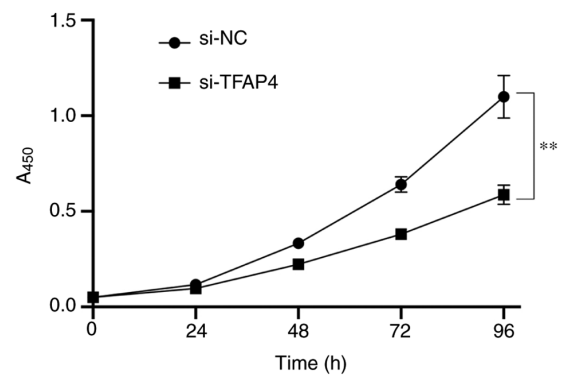

Figure 6. TFAP4 knockdown significantly inhibits the proliferation of SKBR3 cells. ${ }^{* *} \mathrm{P}<0.01$. TFAP4, transcription factor activating enhancer-binding protein 4; si, small interfering; NC, negative control. the proliferation of SKBR3 cells, not only inducing cell cycle arrest, but also leading to cell apoptosis (27) and significantly impairing the migratory and proliferative abilities of SKBR3 tumor cells.

Previous studies have reported that TFAP4 is regulated by other miRNAs. For example, miR-302c suppresses EMT and metastasis by targeting TFAP4 in colorectal cancer (32). In addition, TFAP4 is a direct target of miR-15a/16-1, which is induced by p53 (33). In the present study, TFAP4 was identified as a direct target of miR-124 in SKBR3 cells. Overexpression of miR-124 significantly attenuated the migratory and proliferative abilities of SKBR3 cells by downregulating TFAP4 
expression. Taken together, these results suggest that miR-124 exerts anti-metastatic and anti-proliferative roles in SKBR3 cells by downregulating TFAP4 expression.

The present study had limitations. The present study only evaluated the function of miR124/TFAP4 axis in Her2+ breast cancer cell line SKBR3. The function of this regulatory mechanism in HER 2- breast cancer will be investigated in future studies. In addition, the results of the present study were not verified in clinical samples. Future studies need to perform experiments in clinical samples to verify the findings of the present study.

In conclusion, the results of the present study suggested that miR-124 can attenuate the migration and proliferation of SKBR3 cells by directly downregulating TFAP4 expression. This confirms the anti-metastatic role of miR-124, which may represent a potential candidate for effective treatment of breast cancer.

\section{Acknowledgements}

Not applicable.

\section{Funding}

The present study was supported by the Natural Sciences Foundation of Inner Mongolia (grant no. 2018MS08010).

\section{Availability of data and materials}

The data that support the findings of the present study are available in the CNSA (https://db.cngb.org/cnsa) of CNGBdb, with accession number CNP0001017. The data will be available from this repository one year after initially submission (April 24th, 2021).

\section{Authors' contributions}

$\mathrm{NC}$ performed the cell experiments, prepared the figures and wrote the manuscript. BJ and YH performed the qPCR and western blot analysis and data interpretation. WL, XH and WG performed the bioinformatics analysis. YG and WB designed the study and revised the manuscript. NC and YG confirm the authenticity of all the raw data. All authors read and approved the final manuscript.

\section{Ethics approval and consent to participate}

Although human samples or patient data were not included in the present study, CNSA requires ethics approval when uploading sequencing data. The present study was approved by the Ethics Committee of Inner Mongolia Autonomous Region People's Hospital (Hohhot, China) (approval no. 202001005L).

\section{Patient consent for publication}

Not applicable.

\section{Competing interests}

These authors declare that they have no competing interests.

\section{References}

1. Azamjah N, Soltan-Zadeh Y and Zayeri F: Global trend of breast cancer mortality rate: A 25-year study. Asian Pac J Cancer Prev 20: 2015-2020, 2019.

2. Li M, Marin-Muller C, Bharadwaj U, Chow KH, Yao QZ and Chen CY: MicroRNAs: Control and loss of control in human physiology and disease. World J Surg 33: 667-684, 2009.

3. Oliveto S, Mancino M, Manfrini N and Biffo S: Role of microRNAs in translation regulation and cancer. World J Biol Chem 8: 45-56, 2017.

4. Silber J, Lim DA, Petritsch C, Persson AI, Maunakea AK, Yu M, Vandenberg SR, Ginzinger DG, James CD, Costello JF, et al: miR-124 and miR-137 inhibit proliferation of glioblastoma multiforme cells and induce differentiation of brain tumor stem cells. BMC Med 6: 14, 2008

5. Chen J, Xiao H, Huang Z, Hu Z, Qi T, Zhang B, Tao X and Liu SH: MicroRNA124 regulate cell growth of prostate cancer cells by targeting iASPP. Int J Clin Exp Pathol 7: 2283-2290, 2014.

6. Hu CB, Li QL, Hu JF, Zhang Q, Xie JP and Deng L: miR-124 inhibits growth and invasion of gastric cancer by targeting ROCK1. Asian Pac J Cancer Prev 15: 6543-6546, 2014.

7. Ma T, Zhao Y, Wei K, Yao G, Pan C, Liu B, Xia Y, He Z, Qi X, Li Z, et al: MicroRNA-124 functions as a tumor suppressor by regulating $\mathrm{CDH} 2$ and epithelial-mesenchymal transition in non-small cell lung cancer. Cell Physiol Biochem 38: 1563-1574, 2016.

8. Shi P, Chen C, Li X, Wei Z, Liu Z and Liu Y: MicroRNA124 suppresses cell proliferation and invasion of triple negative breast cancer cells by targeting STAT3. Mol Med Rep 19: 3667-3675, 2019.

9. Ji H, Sang M, Liu F, Ai N and Geng C: miR-124 regulates EMT based on ZEB2 target to inhibit invasion and metastasis in triple-negative breast cancer. Pathol Res Pract 215: 697-704, 2019.

10. Han ZB, Yang Z, Chi Y, Zhang L, Wang Y, Ji Y, Wang J, Zhao H and Han ZC: MicroRNA-124 suppresses breast cancer cell growth and motility by targeting CD151. Cell Physiol Biochem 31: 823-832, 2013.

11. Yan G, Li Y, Zhan L, Sun S, Yuan J, Wang T, Yin Y, Dai Z, Zhu Y, Jiang Z, et al: Decreased miR-124-3p promoted breast cancer proliferation and metastasis by targeting MGAT5. Am J Cancer Res 9: 585-596, 2019.

12. Du S, Li H, Sun X, Li D, Yang Y, Tao Z, Li Q and Liu K: MicroRNA-124 inhibits cell proliferation and migration by regulating SNAI2 in breast cancer. Oncol Rep 36: 3259-3266, 2016.

13. Wang Y, Chen L, Wu Z, Wang M, Jin F, Wang N, Hu X, Liu Z, Zhang CY, Zen K, et al: miR-124-3p functions as a tumor suppressor in breast cancer by targeting CBL. BMC Cancer 16: 826, 2016.

14. Feng T, Xu D, Tu C, Li W, Ning Y, Ding J, Wang S, Yuan L, Xu N, Qian K, et al: miR-124 inhibits cell proliferation in breast cancer through downregulation of CDK4. Tumour Biol 36: 5987-5997, 2015.

15. Li L, Luo J, Wang B, Wang D, Xie X, Yuan L, Guo J, Xi S, Gao J, Lin X, et al: Microrna-124 targets flotillin-1 to regulate proliferation and migration in breast cancer. Mol Cancer 12: 163, 2013.

16. Li W, Zang W, Liu P, Wang Y, Du Y, Chen X, Deng M, Sun W, Wang L, Zhao G and Zhai B: MicroRNA-124 inhibits cellular proliferation and invasion by targeting Ets-1 in breast cancer. Tumour Biol 35: 10897-10904, 2014.

17. Cai WL, Huang WD, Li B, Chen TR, Li ZX, Zhao CL, Li HY, Wu YM, Yan WJ and Xiao JR: microRNA-124 inhibits bone metastasis of breast cancer by repressing Interleukin-11. Mol Cancer 17: 9, 2018.

18. Trapnell C, Roberts A, Goff L, Pertea G, Kim D, Kelley DR, Pimentel H, Salzberg SL, Rinn JL and Pachter L: Differential gene and transcript expression analysis of RNA-seq experiments with TopHat and Cufflinks. Nat Protoc 7: 562-578, 2012.

19. Livak KJ and Schmittgen TD: Analysis of relative gene expression data using real-time quantitative PCR and the 2(-Delta Delta $\mathrm{C}(\mathrm{T})$ ) method. Methods 25: 402-408, 2001.

20. Trapnell C, Williams BA, Pertea G, Mortazavi A, Kwan G, van Baren MJ, Salzberg SL, Wold BJ and Pachter L: Transcript assembly and quantification by RNA-Seq reveals unannotated transcripts and isoform switching during cell differentiation. Nat Biotechnol 28: 511-515, 2010. 
21. Love MI, Huber W and Anders S: Moderated estimation of fold change and dispersion for RNA-seq data with DESeq2. Genome Biol 15: 550, 2014

22. Lin J, Wen X, Zhang X, Sun X, Yunzhi L, Peng R, Zhu M, Wang M, Zhang Y, Luo W, et al: miR-135a-5p and miR-124-3p Inhibit Malignancy of Glioblastoma by Downregulation of Syndecan Binding Protein. J Biomed Nanotechnol 14: 1317-1329, 2018.

23. Lee SU, Song HO, Lee W, Singaravelu G, Yu JR and Park WY: Identification and characterization of a putative basic helix-loop-helix (bHLH) transcription factor interacting with calcineurin in C. elegans. Mol Cells 28: 455-461, 2009.

24. Li X, Liang L, Huang L, Ma X, Li D and Cai S: High expression of protein phosphatase 4 is associated with the aggressive malignant behavior of colorectal carcinoma. Mol Cancer 14: 95, 2015.

25. Liu X, Zhang B, Guo Y, Liang Q, Wu C, Wu L, Tao K, Wang G and Chen J: Down-regulation of AP-4 inhibits proliferation, induces cell cycle arrest and promotes apoptosis in human gastric cancer cells. PLoS One 7: e37096, 2012

26. Cancer Genome Atlas Network: Comprehensive molecular characterization of human colon and rectal cancer. Nature 487: 330-337, 2012.

27. Wang YF, Ao X, Liu Y, Ding D, Jiao WJ, Yu Z, Zhai WX, Dong SH, He YQ, Guo H and Wang JX: MicroRNA-608 promotes apoptosis in non-small cell lung cancer cells treated with doxorubicin through the inhibition of TFAP4. Front Genet 10: 809, 2019

28. Huang T, Chen QF, Chang BY, Shen LJ, Li W, Wu PH and Fan WJ: TFAP4 promotes hepatocellular carcinoma invasion and metastasis via activating the PI3K/AKT signaling pathway. Dis Markers 2019: 7129214, 2019.
29. Torre LA, Bray F, Siegel RL, Ferlay J, Lortet-Tieulent J and Jemal A: Global cancer statistics, 2012. CA Cancer J Clin 65: 87-108, 2015.

30. Song J, Xie C, Jiang L, Wu G, Zhu J, Zhang S, Tang M, Song L and $\mathrm{Li} \mathrm{J}$ : Transcription factor AP-4 promotes tumorigenic capability and activates the $\mathrm{Wnt} / \beta$-catenin pathway in hepatocellular carcinoma. Theranostics 8: 3571-3583, 2018.

31. D'Annibale S, Kim J, Magliozzi R, Low TY, Mohammed S, Heck AJ and Guardavaccaro D: Proteasome-dependent degradation of transcription factor activating enhancer-binding protein 4 (TFAP4) controls mitotic division. J Biol Chem 289: 7730-7737, 2014.

32. Ma W, Liu B, Li J, Jiang J, Zhou R, Huang L, Li X, He X and Zhou Q: MicroRNA-302c represses epithelial-mesenchymal transition and metastasis by targeting transcription factor AP-4 in colorectal cancer. Biomed Pharmacother 105: 670-676, 2018.

33. Shi L, Jackstadt R, Siemens H, Li H, Kirchner T and Hermeking H: p53-induced miR-15a/16-1 and AP4 form a double-negative feedback loop to regulate epithelial-mesenchymal transition and metastasis in colorectal cancer. Cancer Res 74: 532-542, 2014.

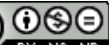

This work is licensed under a Creative Commons Attribution-NonCommercial-NoDerivatives 4.0 International (CC BY-NC-ND 4.0) License. 